\title{
Comparing choices and variations in people and rats: Two teaching experiments
}

\author{
ALLEN NEURINGER, CHRIS DEISS, and SCOTT IMIG \\ Reed College, Portland, Oregon
}

\begin{abstract}
Two pairs of experiments enabled students to compare their own operant behaviors with those of rats. The students played computer games for points, and the rats pressed levers for food. The first pair of experiments showed that, under concurrent schedules of reinforcement, relative frequencies of choices between two alternatives increased linearly in rats and people as functions of relative frequencies of reinforcement, with similar biases and undermatching observed in both species. The second pair of experiments showed that behavioral variability was controlled by reinforcers contingent on variability, this again true for both species. These experiments helped demonstrate the relevance of animal operant research to an explanation of human operant behavior.
\end{abstract}

Introductory psychology at Reed College is a broadly based year-long course combining lectures, conferences, and research in all major areas of psychology. One section at the beginning of the second semester focused on operant behavior. An issue for students is whether studying animals is relevant to an explanation of human actions. To enable students to compare human and rat behaviors, we developed two pairs of procedures in which rats pressing levers for food paralleled students playing computer games for points. Prior to playing the games, the students were not informed of the relationship between the rat and human procedures.

We wanted the students to accomplish three goals: to study the influences of reinforcement contingencies, to compare their own behaviors to those of rats, and, because the students were themselves subjects, to evaluate how their subjective evaluations of the contingencies were related to the actual contingencies. During 30 years of teaching introductory psychology, the senior author has found no better way to demonstrate the importance of studying the operant responses of animal models.

\section{EXPERIMENT 1A Concurrent Choice in Rats}

The study of choices by animals constitutes a major area of research (Williams, 1988). Under the commonly

This research was supported in part by grants from the National Science Foundation to A.N. The authors thank Gene Olson for excellent animal care and a helpful reading of the manuscript. All programs were written in True Basic and can be obtained (for use with Macintosh computers) from the authors. The human programs can be downloaded from $\mathrm{ftp}$ //ftp.reed.edu/reed/projects/neuringer/. The rat programs require specialized interfaces and operant equipment. Correspondence and requests for the two computer games (for use with Macintosh computers) should be addressed to A. Neuringer, Psychology Department, Reed College, Portland, OR 97202 (e-mail: allen_neuringer@reed.edu). used "concurrent reinforcement" procedure, a rat or pigeon is presented with two alternatives, each associated with a different reinforcement rate. A matching relationship is sometimes observed, showing that the proportion of choices between the two operanda (e.g., left and right levers) equals the proportion of reinforcements obtained for responding on the two levers (Baum, 1974; Herrnstein, 1961). For example, if $30 \%$ of the reinforcers are obtained from the left lever, then $30 \%$ of choice responses are made to that lever. More common is undermatching, where the proportion of choices changes more slowly than the proportion of reinforcers (i.e., choices are closer to $50 \%$ than predicted by the reinforcers; Baum, 1974). Most important, choices show an orderly relationship to reinforcement rates.

In most experiments, each subject chooses between many pairs of reinforcement rates programmed by variable-interval (VI) schedules, with as many as 30 sessions or more devoted to each pair. Because Experiment $1 \mathrm{~A}$ took place in a single laboratory period in which 81 students experimented on 40 rats, procedural changes were required. First, each rat was randomly assigned only one pair of schedules, with different rats experiencing different pairs. Second, fixed-interval (FI) schedules were used rather than the more common VIs (Shimp, 1971, also used concurrent FIs). FI values were easier for students to modify on line (see below), and, in addition, the rats experienced the schedule for about $35 \mathrm{~min}$ total time. Third, because the rats had little previous training, the student experimenters were permitted to change (within the experimental session) the absolute frequencies of reinforcement, so as to maintain high response strengths in their individual rats. For a given subject, however, the ratio of reinforcement frequencies was fixed and constant throughout. The goal of the laboratory was to determine, within a single 35 -min session, whether choices by rats were related in orderly fashion to relative rates of reinforcement and, if so, the form of the function. 


\section{Method}

Laboratory. The research took place in the Animal Core Lab at Reed College, a student laboratory containing 10 operant chambers in 10 cubicles, each of which opened onto a central common space. Two students usually worked together in one cubicle. The Animal Colony, in which the rats were housed, was located about $25 \mathrm{ft}$ from the Lab, and most students carried the rats back and forth from Colony to Lab. Instruction was given concerning care and handling of rats and ethical issues. (Two of the 83 students enrolled were given alternative assignments because they chose not to engage in research involving animals.)

Subjects. Forty Long-Evans male rats, approximately 90 days old, were shaped by the students to press the right of two levers in an operant chamber (first 35 -min session), then received $10 \mathrm{~min}$ of extinction of right-lever presses, and were shaped to press the left lever (second session). In both sessions, after the rat had learned to press the appropriate lever, the students could increase the fixed number of responses required for each pellet, with a ratio of 5 responses/pellet rarely exceeded. The present experiment took place during the rats' third session in the operant chamber. The rats were maintained on a 21 to 22 -h food-deprivation regimen throughout, with $2 \mathrm{~h}$ of free food provided immediately following each session. Water was always available in the home cages, and the rats lived in same-sex pairs. A 12:12-h light:dark cycle was maintained.

Apparatus. Each of 10 Gerbrands operant chambers $(26.7 \times$ $27.9 \times 29.2 \mathrm{~cm}$ ) contained two response levers, $5.1 \mathrm{~cm}$ wide and $8.9 \mathrm{~cm}$ apart, located on the right side wall and on either side of a food receptacle into which 45-mg Noyes food pellets could be delivered as reinforcers. Above each lever was a white light. An overhead light could illuminate the chamber, and a speaker could provide auditory stimuli. Each chamber was contained in a light- and sound-attenuating box and was controlled by a Macintosh Classic II computer with the True BASIC programming language through a MetaResearch interface module.

Procedure. Two FI schedules operated concurrently: one controlling availability of reinforcers contingent on left-lever responses, and the other controlling availability of reinforcers contingent on right-lever responses. When a fixed interval had elapsed, the next response to the associated lever produced a food pellet and restarted the interval, with a scheduled reinforcement being "held" until the rat responded on the lever. The two schedules were completely independent--responding on one lever had no influence on reinforcers available from the other lever-and there was no penalty for switching between the two schedules (i.e., no changeover delay, COD).

Each rat was randomly assigned one of four relative frequencies of reinforcement, with the ratio of $[L /(L+R)]$ reinforcement frequencies (reinforcements/sec) being .14, $.25, .5$, and .75 (i.e., in the last case, the right $\mathrm{FI}-\mathrm{sec} /$ reinforcement-was three times longer than the left). Once assigned at the beginning of the session, these ratios could not be changed. However, the student experimenters could modify the absolute value of the left FI, this automatically determining the value of the right FI according to the just enumerated ratios. Written instructions to the student experimenters included the following:

Since we are interested in obtaining a stable index of choice .... you will be able to vary the absolute values of the fixed intervals. If these intervals are too short (e.g., $0.1 \mathrm{sec}$ and $0.3 \mathrm{sec}$ ), there would be no reason for the rat ever to vary its responses. If the intervals were too long, (e.g., $10 \mathrm{~min}$ and $30 \mathrm{~min}$ ), responding would extinguish. Thus, your task will be to continually change the absolute values of the intervals, if this is necessary, in order to enable your rat to demonstrate its preference, and the degree of that preference.

Plotted on the computer screen were cumulative relative frequencies of responses (left/left+right) on the $y$-axis, as a function of time on the $x$-axis. The students were told not to attempt to generate any particular ratio of choices but rather to attempt to generate suffi- ciently high response rates and orderly data, by manipulating absolute reinforcement values, so as to estimate the rat's preference. Thus, students observed ratios of left to right responses emerging across the session and, when necessary, increased (if responding was slowing) or decreased (if too many pellets were being eaten) absolute reinforcement frequencies. The session lasted approximately $35 \mathrm{~min}$.

\section{Results}

Data from the last $10 \mathrm{~min}$ of the session were used to represent performance. Figure 1 (top panel) shows the proportion of left responses (left/left+right) as a function of the proportion of reinforcers obtained for responding on the left lever, each data point representing 1 of the 40 rats. The least squares line accounted for $79 \%$ of the variance $\left(r^{2}=.79\right)$, with a slope of 0.69 indicating undermatching, and a $y$-intercept of 0.19 indicating a bias for the left lever.

Because reinforcement was frequent, distributions of choices might have influenced the distributions of reinforcements, rather than vice versa. ${ }^{\prime}$ Figure 2 (top panel) shows that this problem did not occur, since obtained relative frequencies closely approximated the programmed reinforcement frequencies, with $96 \%$ of the variance described by the least squares line, the slope of the bestfitting line approaching 1.0, and an intercept close to zero.

Figure 3 shows the proportion of left responses, summed across the preceding period of the session, at each minute of the first $25 \mathrm{~min}$ of the session (the minimum session length for all subjects). Group averages at each of the relative reinforcement frequencies $(.14, .25, .5$, and .75) show a strong initial left-response bias, with stable averages by the end of the session, although continuing to be biased toward the left.

\section{Discussion}

A linear relationship between relative rates of responding (choices) and relative rates of reinforcement was obtained from 40 rats in one 35 -min session. A bias for the left lever was probably due to the fact that, in the previous session, right-lever pressing was extinguished and left presses conditioned. Also found was undermatching (slope of the least squares line less than 1.0), indicating more equal distribution of choices across the two levers than predicted by the matching law. Most important for its use in an educational setting, the procedure showed rapid adaptation by relatively naive rats to the schedules of reinforcement and an orderly relationship between choices and consequences. (See Gallistel, 1990, for other examples of rapid adaptation to concurrent reinforcement schedules.)

There were a number of ways in which the present procedure differed from those commonly employed. First, absolute rates of reinforcement were varied by the students, this being done within the experimental sessions, whereas relative reinforcement rates were held constant. Generally, reinforcement schedules are constant across many sessions. Second, the matching relationship was shown by aggregating data from 40 different subjects, each 

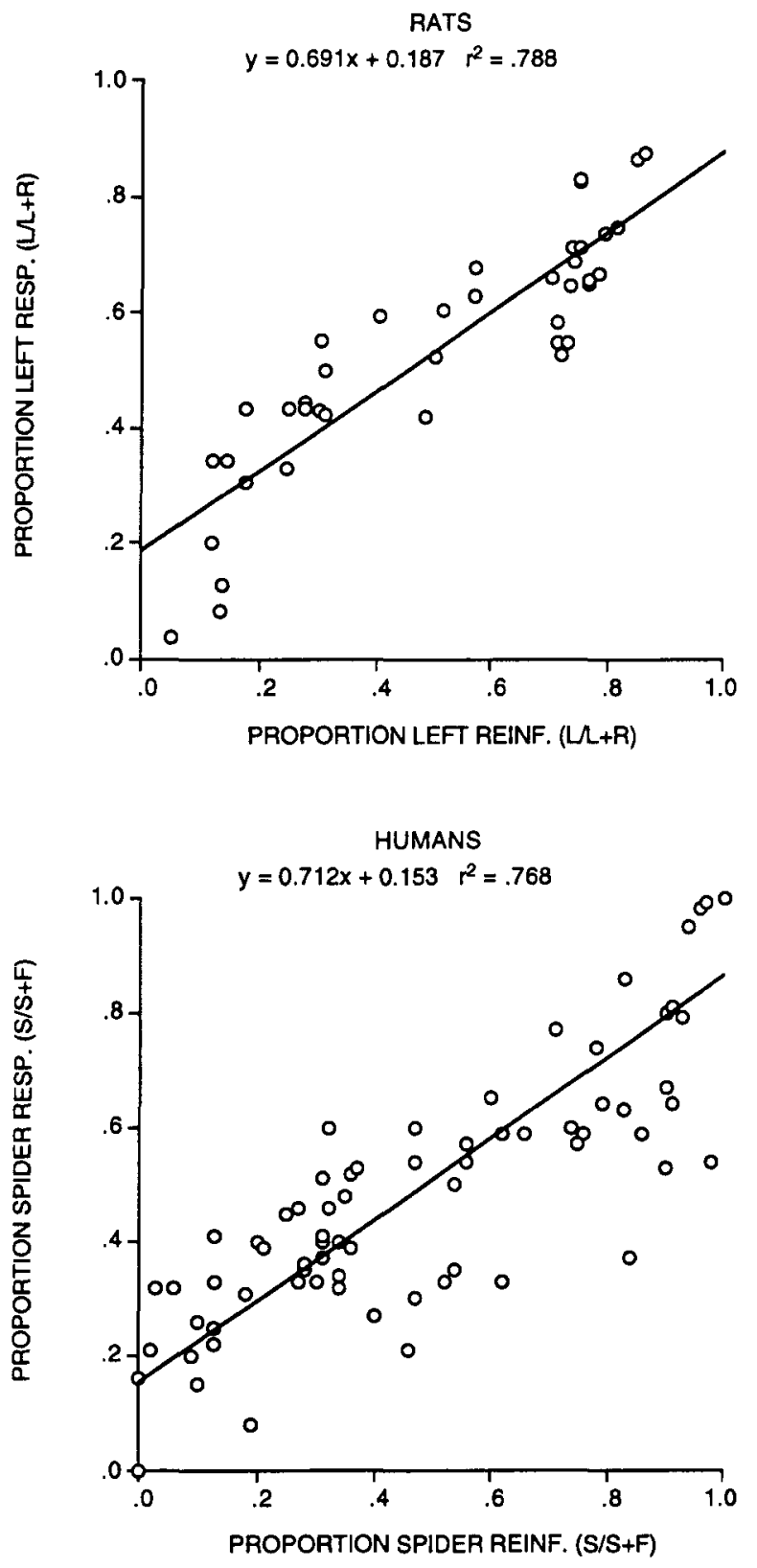

Figure 1. Top: Proportion of left-lever presses by rats (left/ left+right) as a function of proportion of obtained left reinforcers. Bottom: Proportion of space-bar responses by human subjects directed at spiders (spider/spider+fly) as a function of proportion of reinforcements obtained for capturing the spiders. Least squares best-fitting lines are drawn in both cases.

having experienced a single ratio of reinforcement. In most previous cases, matching was demonstrated using withinsubject comparisons, where each subject experiences many different reinforcement ratios. Third, the entire experiment was accomplished within a single 50 -min class period, with approximately 35 min devoted to the study. Generally, many months are required. Each of these was mandated by teaching constraints. However, the results are consistent with those from the research literature (Davison \& McCarthy, 1988). This single-session demonstration of a matching-like relationship in rats led to class discussion of the meaning of "choice" and how choices were influenced by reinforcers.

\section{EXPERIMENT 1B Concurrent Choice in People}

In Experiment 1B, students' choices were studied. The students sat at the same computer used in Experiment $1 \mathrm{~A}$ and, by controlling the movement of a frog icon on the computer screen, attempted to capture as many flies (one choice) or spiders (the other choice) as possible. Unbeknownst to the students, whether a spider or a fly could be captured depended on whether an associated VI schedule of reinforcement had timed out (i.e., had made reinforcement available). Five different relative reinforcement frequencies were randomly distributed across the human subjects, the main questions being the same as for the rats in Experiment 1A. At the end of the session, the students were asked to estimate how frequently they attempted to capture flies and spiders, to estimate how frequently they were reinforced for each, and to describe their strategies. These verbal estimates were compared with their actual responses in order to compare thoughts about (or verbalizations concerning) performance with performance itself. In brief, just as the students had studied rat choices, their own choices were now studied as a function of relative frequencies of reinforcement.

\section{Method}

Subjects. The 81 students who had served as experimenters in Experiment $1 \mathrm{~A}$ were the subjects.

Apparatus. The Macintosh Classic II computers used in Experiment $1 \mathrm{~A}$, with black-and-white monitors $(173 \times 115 \mathrm{~mm})$, were again used. Programs were again written in True BASIC. The subjects entered responses on the keyboard, received visual feedback on the screen, and heard auditory feedback from the computer's speaker.

Procedure. During one 50-min laboratory period, each student played two computer "games," one of which constituted the current experiment. The actual order of the "running" of the experiments was $1 \mathrm{~A}, 2 \mathrm{~A}, 1 \mathrm{~B}$, and $2 \mathrm{~B}$ for half the subjects and $1 \mathrm{~A}, 2 \mathrm{~A}, 2 \mathrm{~B}$, and $1 B$ for the other half. Thus, the computer-game procedures followed completion of the rat procedures. For purposes of exposition, we pair the rat and human procedures.

On the screen was a small frog ( $7 \mathrm{~mm}$ wide $\times 6 \mathrm{~mm}$ high) and a variable number of randomly moving spider symbols (in one of two schedule components) or randomly moving fly symbols (in the alternate component), each $9 \mathrm{~mm}$ wide $\times 4 \mathrm{~mm}$ high. Pressing the "<" or "> key caused the frog to rotate counterclockwise or clockwise, respectively. Pressing the "L" key caused it to move straight ahead. Pressing the space bar caused the frog's tongue to "shoot out" until it hit a spider or a fly, thereby effecting a "capture," or missed and reached the edge of the screen. Pressing the "X" key caused a change from the fly component to the spider component, or vice versa (i.e., the " $X$ " key served as a toggle between the two schedule components under a changeover-key procedure; Findley, 1958). Each capture of a fly or a spider added 100 points to the score, flies and spiders being equally valuable. All responses to the space bar and the " $X$ " key subtracted 2 points, analogous to 

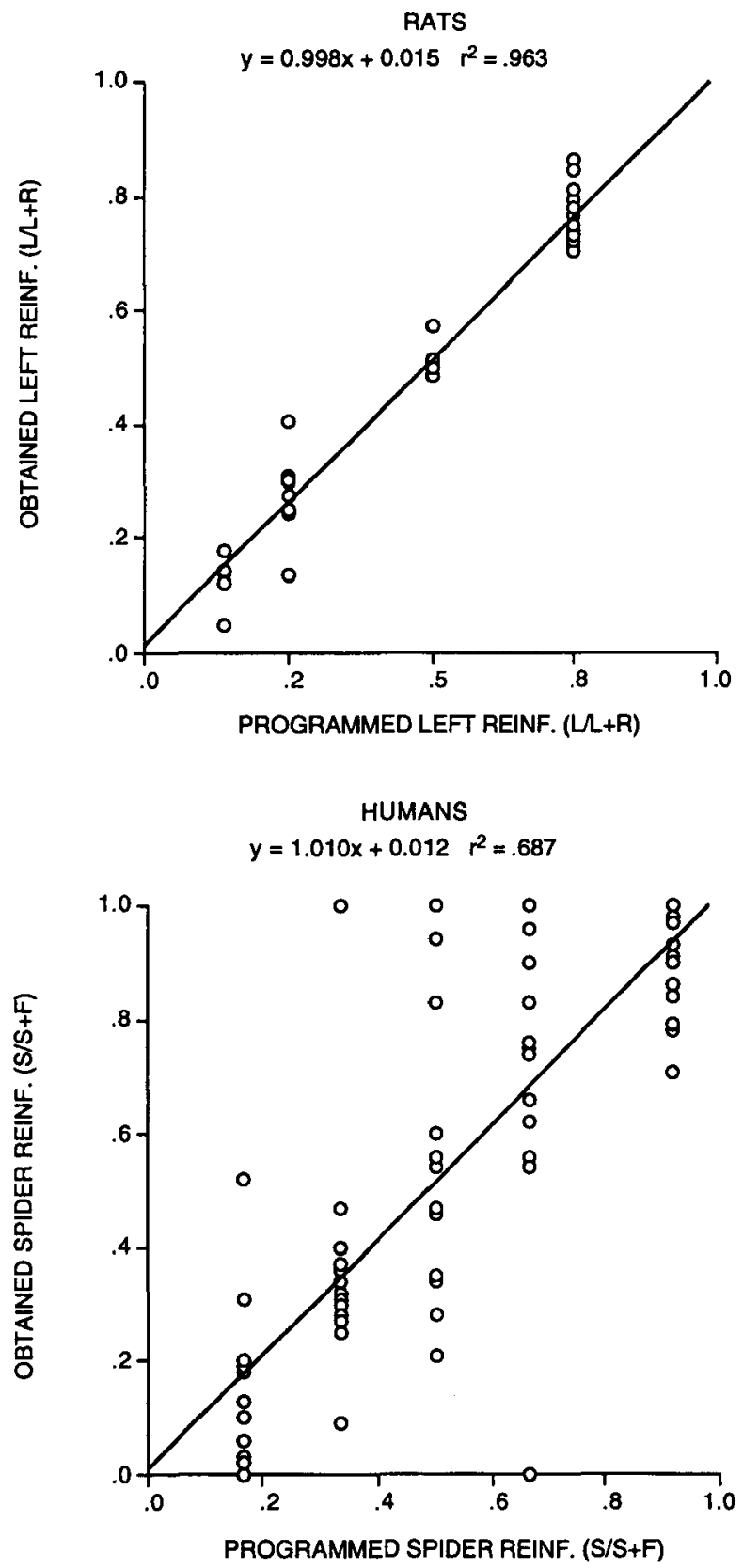

Figure 2. Top: Proportion of reinforcements obtained by rats for responding on the left lever as a function of the programmed proportion of left reinforcements. Bottom: Proportion of reinforcements obtained by human subjects for shooting at spiders as a function of the programmed proportion of spider reinforcements. Least squares best-fitting lines are drawn in both cases.

the effort taken to press levers by the rats, and, as for the rats, there was no differential penalty for switching. The current score was visible at the bottom of the screen. Sessions lasted $15 \mathrm{~min}$, and the subjects were told that those with the two highest scores in each laboratory section would be invited to dinner prepared by the senior author (A.N.).

Randomly allocated across the students were five pairs of variable interval schedules, the first number of the pair indicating average interteinforcement interval (in seconds) in the spider component, the second indicating the average in the fly component, with relative frequencies (spider/spider+fly) in parentheses: VI $20-\mathrm{VI}$ 20 (.50), VI 15-VI 30 (.67), VI 30-VI 15 (.33), VI 60-VI 12 (.17), and VI 10.9-VI $120(.92)$. Overall programmed frequencies of reinforcement - reinforcements summed across both alternativeswere 6 per min for each pair of reinforcement schedules. Each of the VI schedules was composed of five randomly ordered intervals generated from a logarithmic function. Both fly and spider VIs ran continuously, whether or not the subject was in the associated component. When one of the intervals elapsed, a potential reinforcement was added to the appropriate "stack" such that the next effective space-bar response in that component would be reinforced (a fly or a spider would be captured and 100 points gained). There was a minimum interval between consecutive reinforcements within a component averaging $2.5 \mathrm{sec}$ (range .5 to $5 \mathrm{sec}$ ), in order to avoid stacked reinforcements being collected for consecutive responses.

Thus, a response was recorded whenever the space bar was pressed, whether or not the frog was facing the potential prey and whether or not a reinforcement was received. However, to be reinforced, the frog had to be facing in the general direction of the potential prey, and the variable interval governing reinforcements had to have elapsed. Since the flies and spiders moved around in quasirandom fashion, jumping up and down and left and right, responses directed toward them before a time for reinforcement had elapsed appeared quite naturally to miss the target. It was not likely, therefore, that the subjects could discriminate why a response at one time would miss but, at another time, would hit-be reinforced - the jumpily moving prey object.

One additional aspect of the game helped to maintain rapid responding: Flies and spiders moved toward the frog, and, if they made contact, the frog was said to be "eaten" and 50 points were lost. To avoid being eaten, the frog could be moved away from approaching bugs, although, as bugs were captured by the frog, their speed of movement increased slightly, making evasion more diffi* cult. Whenever the frog was "eaten," bug speed decreased. An alternative way to protect the frog was to shoot the tongue (press the space bar) or rotate (press the " $<$ " or " $>$ " key), thereby setting up a "force field" (indicated by lines emanating from the frog): When the force field was activated, the frog could not be eaten. The chances of being eaten were identical in fly and spider components.

At the end of the experiment, the subjects were asked to estimate the number of space-bar responses over the last $5 \mathrm{~min}$ of the experiment in spider and fly components, separately, and the number of captures (reinforcements) attained in each component. They were also asked to "describe your strategy for getting points." These estimations were entered at the computer terminal and were later used to compare with the subjects' behaviors.

In summary, human subjects chose between two alternatives, responding to flies and to spiders. The data of interest were numbers of "shots" (i.e., responses on the space bar) in the fly environment versus in the spider environment. Space-bar responses to spider and fly were therefore analogous to left- and right-lever presses by rats. The game stopped when $15 \mathrm{~min}$ had elapsed.

\section{Results}

Data analyses were based on the last 5 min of the 15 min session. Figure 1 (bottom panel) shows the proportion of space-bar responses in the spider component (spider/ spider + fly space-bar responses) as a function of the proportion of obtained reinforcers (spider/spider+fly captures). The function was similar to that obtained from the rats, with a slope of 0.71 , intercept of 0.15 , and $r^{2}$ of .77 . As with the rats, undermatching was observed as well as a bias for one of the alternatives (in this case, for spiders).

Figure 2 (bottom panel) shows that obtained relative frequencies of reinforcement approximated the programmed relative frequencies (slope of the function was 


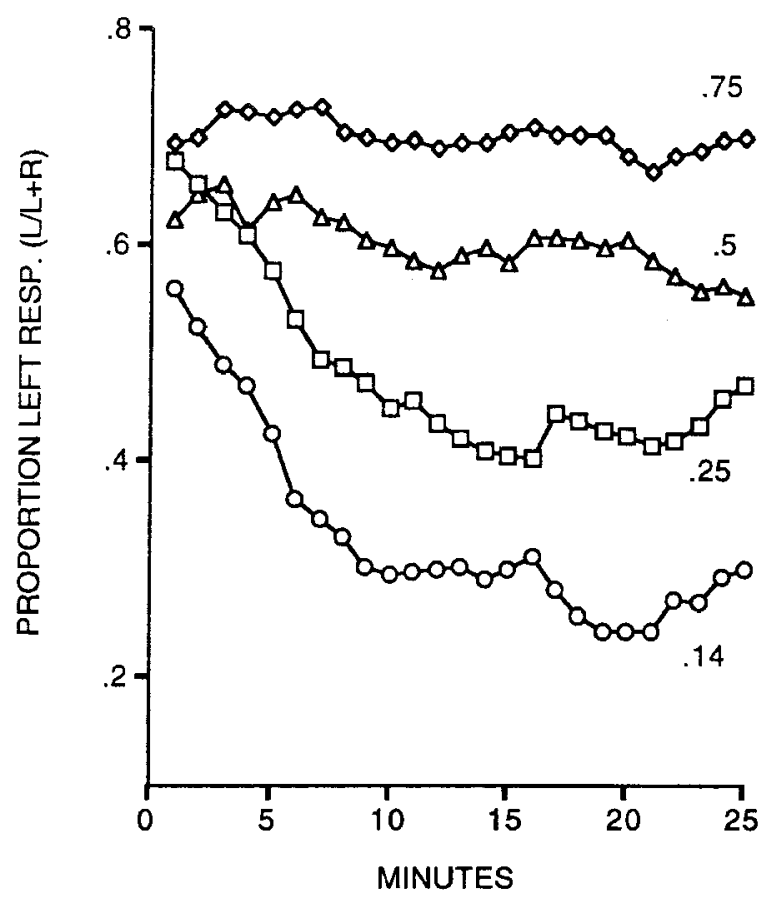

Figure 3. Proportion of left responses as a function of time in the session for each the four programmed proportions of reinforcements (left/left+right).

1.01 ) with little bias ( $y$-intercept was 0.01$)$, both of these parameters paralleling the rat data shown above, but $r^{2}$ was .69 , lower than for the rats.

Figure 4 (top panel) shows that when the students were asked to estimate their responses and captures, the estimated values yielded a function similar to the behavioral data in Figure 1 (bottom panel). The slope was lower (greater degree of undermatching) for the estimations than for the actual responses, the $y$-intercept was higher (more bias for spiders), and goodness of fit was lower. Figure 4 (middle panel) shows the actual choices as a function of the estimates of the frequencies of reinforcement (i.e., the $y$-axis data are the same as in the bottom panel of Figure 1, and the $x$-axis data the same as in the top panel of Figure 4). Both slope (0.88) and $y$-intercept $(0.069)$ were now closer to exact matching than in the cases of either behavioral data alone or estimations alone. That is, actual choices matched estimated reinforcements more closely than they matched actual reinforcements. On the other hand, $r^{2}$ was lower than in the behavioral case.

When asked to describe their strategies, only 1 of the 81 subjects identified the underlying concurrent reinforcement contingencies, despite the fact that concurrent schedules had been discussed in a lecture period preceding this laboratory and the students had used concurrent schedules in the just-preceding laboratory (a few days earlier) on rat choice. Some of the behaviors described were functional or closely related to the underlying contingencies (e.g., frequent switching between the fly and spider components, shooting rapidly when spiders or flies approached, and spending more time in the higher reinforcement component). However, erroneous hypotheses were frequently offered as to how best to gain points. Here are some representative examples, each from a different student (with the language slightly revised for ease of presentation):

move around to trick the spiders and flies; walk in front of the little bug's paths and then shoot; turn to the left before shooting; the closer to the middle of the screen, the better; hit them from the side or top; stay in one place, towards the top center of the screen; stay in the center and revolve continuously; move around a lot; make a full circle on the fly screen; let the flies and spiders get as close as possible to the frog before shooting; position the frog at a $45^{\circ}$ angle to the spider or fly; spin around in a circle and anticipate where the bugs would be, then shoot; get closer and closer and closer to fly or spider and shoot intensely; change angles to deceive the spiders and flies; shoot mainly toward the corners of the screen; wait for them to come to me.

None of these strategies in fact directly influenced reinforcement.

\section{Discussion}

When choices under concurrent schedules of reinforcement are studied in the laboratory, human performance often differs from that of nonhuman animals, but, under naturalistic circumstances, humans behave more like animals (Kollins, Newland, \& Critchfield, 1997; Mazur, 1998). The present experiment supports previous indications that computer games also result in human choices approximating choices observed in nonhuman animals (Allan, 1995).

There were many differences between the procedure used to study rat choices in Experiment $1 \mathrm{~A}$ and that used to study student choices in the Experiment $1 \mathrm{~B}$. Responses, reinforcers, and stimuli differed, as did precise contingencies (concurrent FIs for rats and Findley-concurrent VIs for people) and motivations (food for rats and points for people), among others. Rather than equating attributes of the experimental situation, we attempted to create procedures that would generate orderly data.

The results from students and rats were similar in many ways, including linear functions relating relative choices to relative reinforcement rates, bias, and undermatching. The bias for left responses in the rats could be attributed to the extinction of right-lever presses and reinforcement of left-lever presses in the previous session. The bias for the spiders in the human case is harder to interpret, but some subjects reported that the spiders appeared to be larger than the flies. Although overall sizes of the spiders and flies were objectively identical, the spiders had multiple legs, which may have caused them to appear larger. Although extent and direction of biases are no doubt a function of particular stimuli and procedures, of most interest is that both people and rats showed biases.

Both people and rats also showed undermatching. Other than the minimal energy expended for switching 

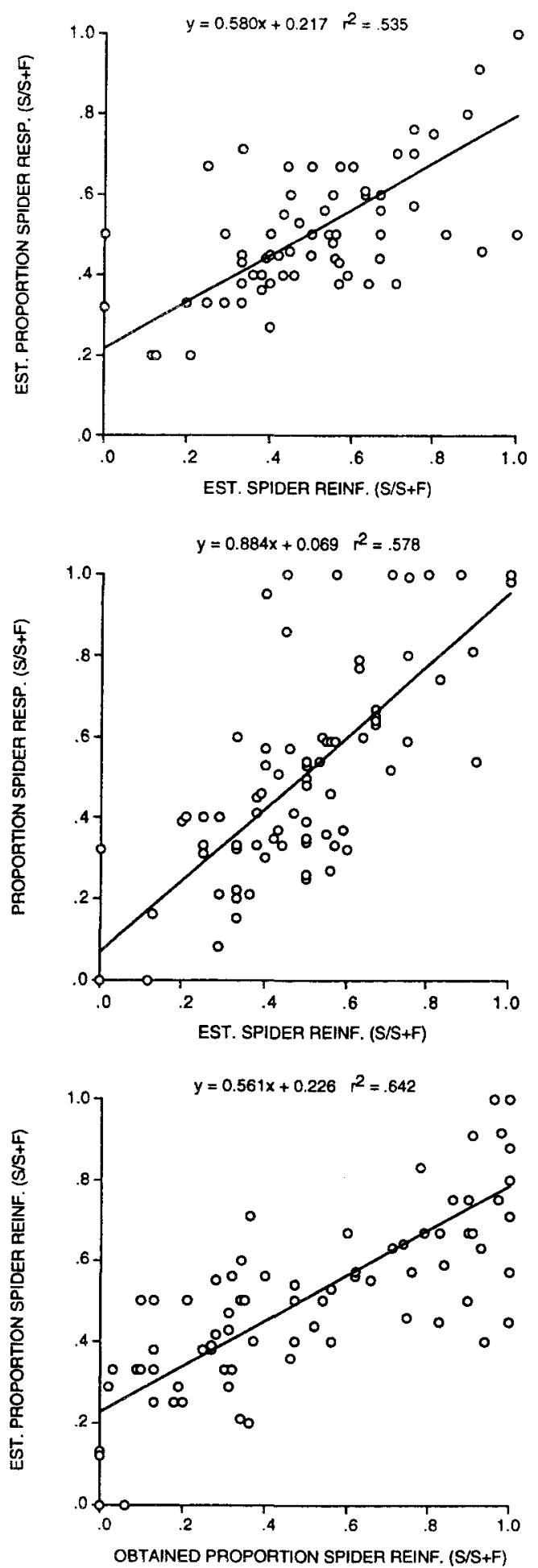

Figure 4. Top: Estimated proportion of spider responses as a function of the estimated proportion of spider reinforcements. Middle: Proportion of actual spider responses as a function of the estimated proportion of spider reinforcements. Bottom: Estimated proportion of spider reinforcements as a function of the proportion of spider reinforcements actually experienced. In all three cases, least squares best-fitting lines are drawn. between the two levers in the case of rats and the loss of minimal points for switching between the two components in the case of students (which was the same as the point loss contingent on all responses), there were no appreciable penalties for switching, and, especially in the human game, switching may have been perceived as functional, momentarily removing a threatening spider or fly. High rates of switching (choosing Alternative 1 followed by Alternative 2, or vice versa) relative to repeating (choosing Alternative 1 followed by another choice of 1 or 2 followed by 2) would lead to undermatching.

The function relating estimates of responses and reinforcers was similar to that relating actual responses and reinforcers. In fact, the closest approximation to matching (equality between relative frequencies of responses and reinforcers) was from actual responses and estimates of reinforcers. Estimates made while subjects engage in an activity may be one source of behavioral undermatching. In support of this hypothesis, Figure 4 (bottom panel) shows relative frequencies of estimated reinforcers as a function of the relative frequencies of obtained reinforcers. Undermatching and bias for spiders are again observed.

The students" "strategies for getting points," as described by them at the end of the experiment, were generally unrelated to the actual reinforcement contingencies. When the contingencies were later described, some students grimaced, groaned, and then laughed. Many indicated surprise that what they had thought was going on was in fact quite different from the true conditions and that these conditions paralleled their recently concluded experiment with rats. That student choices could be predicted by relative frequencies of reinforcement while, at the same time, their explicit hypotheses concerning the contingencies were wrong led to a discussion of the roles of cognition and contingency in choice (see Rachlin, 1989, for an excellent presentation of these issues).

\section{EXPERIMENT 2A Reinforced Variability in Rats}

Response variations can be reinforced (Machado, 1989; Page \& Neuringer, 1985). Such reinforced variability may contribute to problem solving and creativity, topics of interest to many students, as well as to operant learning generally (Denney \& Neuringer, 1998; Stokes, 1999). Commonly studied in the behavioral laboratories are sequences of left and right responses, with variability being significantly higher when it is required for reinforcement than when not. In Experiments $2 \mathrm{~A}$ and 2B, rat and human performances when reinforcement depended on high levels of variability were studied and were compared with performances when reinforcement was given without regard to response variations.

In the rat study (Experiment 2A), four responses across left and right levers constituted one trial, with $16\left(2^{4}\right)$ different possible sequences, or patterns (e.g., LLLL, LLLR, LLRL). Under VarT, or variability threshold contingen- 

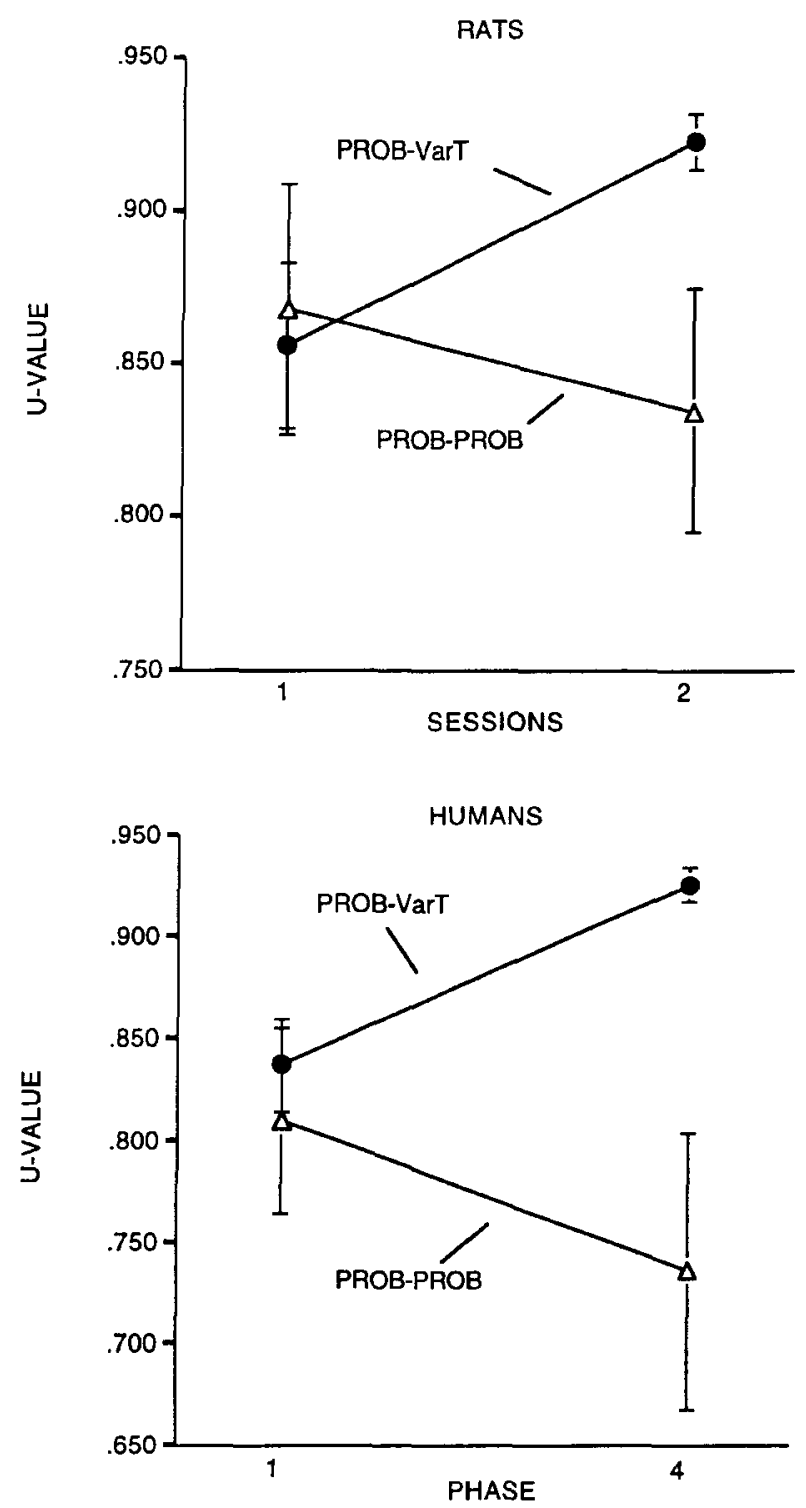

Figure 5. $U$ values, a measure of sequence variability, for rats (top) and human subjects (bottom), as functions of whether reinforcement was contingent on high variability (VarT) or provided independently of variability (Prob). One group in each experiment experienced Prob reinforcement during the first session or phase and Var'T reinforcement during the second session or phase. The other, control, group received Prob reinforcement during both sessions or phases. Standard deviations are shown by the error bars.

cies, a trial terminated with food only if an infrequently occurring sequence had been emitted -our operational definition of high variability.

\section{Method}

Subjects and Apparatus. The rats and the apparatus were the same as in Experiment 1 A, with the two sessions of the present experiment following Experiment $1 \mathrm{~A}$.

Procedure. Each of the first three responses in the trial caused the keylight over the responded-to lever to flash off (become dark) for $0.1-\mathrm{sec}$; the fourth response terminated the trial with either reinforcement or timeout (TO). Reinforcement was the same 45-mg Noyes food pellet used in Experiment $1 \mathrm{~A}$ followed by 0.5 -sec darkness. The TO consisted of only the $0.5 \mathrm{sec}$ of darkness.

In the first of two sessions, whether a trial ended with reinforcement or TO depended only on a probability generator (i.e., consequences did not depend on sequence variations). For half of the rats (arbitrarily chosen), $50 \%$ of the trials terminated with reinforcement; for the remaining rats, $67 \%$ of the trials terminated with reinforcement. Under these probabilistic (Prob) contingencies, sequence variability was permitted but not required.

Prior to the second session, the rats were randomly divided into two groups. Most (33) experienced a variability threshold contingency (VarT) in which high levels of sequence variation were required for reinforcement. The remaining 7 subjects served as controls and experienced the same Prob contingencies as in Session 1. This design permitted us to make both within- and between-subjects comparisons.

Under VarT contingencies, only infrequently occurring sequences were reinforced (Denney \& Neuringer, 1998). Seventeen counters kept track of the sequence frequencies, one for each of the possible 16 sequences and one for their sum. Whenever a sequence occurred, 1 was added to the counter corresponding to that sequence and to the counter corresponding to the sum. To determine whether reinforcement would be provided, the value in the counter corresponding to the just-emitted sequence was divided by the sumsequence counter. If the resulting relative frequency was less than a threshold value, set equal to 0.1 , then a pellet was provided. If the current sequence had been emitted more frequently than $10 \%$ of the time (relative frequency greater than 0.1 ), then TO was given. In this manner, only infrequently occurring sequences were reinforced. After every reinforced trial, the values in all 17 counters were multiplied by a weighting coefficient set equal to .95 . Application of this weighting coefficient resulted in an exponential decrease in the contributions of sequences that had occurred earlier in the session: The further back a particular sequence had occurred, the greater number of times the weighting coefficient had decreased its contribution to the current value. In brief, only sequences that had been emitted less than $10 \%$ of the time over the relatively recent past were reinforced. Sessions terminated after approximately $35 \mathrm{~min}$.

\section{Results}

To assess variability, $U$ values were computed as follows:

$$
-\sum_{i=1}^{16} \frac{\left[\mathrm{RF}_{i} * \log _{2}\left(\mathrm{RF}_{i}\right)\right]}{\log _{2}(16),}
$$

where $\mathrm{RF}_{i}$ refers to the relative frequency of occurrence of each of the 16 sequences. A high $U$ value ( 1.0 being maximum) indicates that each of the 16 possible sequences occurred with a relatively equal distribution. Lower $U$ values ( 0 being the minimum) indicate that one or more sequences had high frequencies, whereas others tended not to occur.

Figure 5 (top panel) shows $U$ values during the first session, in which all rats had received reinforcements probabilistically, and during the second session, in which most of the subjects were reinforced for variable sequences (VarT), while others continued under Prob. ( $U$ value analyses require large quantities of data, and, therefore, we utilized responses from the complete $35-\mathrm{min}$ session.) An analysis of variance showed a significant group $\times$ session interaction $[F(1,38)=4.59, p<.05]$. 
Variability was higher when it was reinforced than when not, this being indicated in two ways. First, the ProbVarT group responded more variably in Session 2 (when variability was reinforced) than it did in Session 1 (when variability was not required) $[F(1,38)=11.77, p<.001]$. Second, the Prob-VarT group responded more variably than did the Prob-Prob group during Session 2 (when only the Prob-VarT group was reinforced for variability) $[F(1,38)=11.59, p<.002]$. Variability in the Prob-Prob group decreased from Session 1 to Session 2, although the decrease did not reach statistical significance. These data show that behavioral variability was controlled by reinforcers contingent on variability.

\section{EXPERIMENT 2B Reinforced Variability in People}

In Experiment 2B, the same question was asked as in Experiment 2A, but with the students serving as subjects. As in Experiment 1B, we attempted to create a situation functionally similar to that provided the rat subjects but differing in the particulars. A computer game was again utilized.

\section{Method}

Laboratory. The laboratory room used for this experiment was normally used for human research and contained, along the outside walls of the room, 12 computers. Students sat in front of the computers and were able to see and hear one another (although they were told to concentrate only on their own "game").

Subjects. The subjects were the same as those in Experiment 1B, except that data from only 71 students are included since a computer malfunction resulted in 10 subjects not participating.

Apparatus. Ten Macintosh computers (variety of models), all with color monitors, were used.

Procedure. The subjects were told to press the " 1 " or " 2 " key to gain points. Unlike in the rat case, responses were emitted continually and without trial demarcation. On the screen was a bar, colored red, which moved up or down following each response. Variable responses caused the bar's height to increase by a constant equal to approximately $3 \%$ of the maximum possible height, whereas responses that did not meet the variability contingency caused the bar to lower by a proportional amount equal to $3 \%$ of its current height. The higher the bar, the more points were allotted per reinforcement-points starting at a +1 level and increasing by increments of 1 to a maximum of +7 per response. The sum of the points gained was shown at the bottom of the bar. At the top edge of the bar was a green line that moved from left to right and served to pace responses with a constant $0.75 \mathrm{sec}$ between consecutive responses. Early and late responses were cued by a two-tone feedback and loss of 3 points. The subjects were told to gain as many total points as possible, and, as in Experiment 1B, those performing best would be invited to A.N.'s house for dinner.

There were four phases, each containing 300 responses. A Prob phase was provided to all subjects during which feedback (points and movement of the red bar) was provided independently of response variability, as was the case for the rats in their Prob phase. Each response had a .5 probability of being positively reinforced.

Without being told, the students were then subdivided into two groups for the remaining three phases. The VarT group was reinforced for varying their response patterns, as will be explained below. The Prob group continued, for the remaining three phases, to be reinforced independently of response variability.
To ensure equality of reinforcement across the two groups, the Prob group's probabilities of reinforcement during Phases 2, 3, and 4 equaled those generated by randomly selected subjects in the VarT group. If a VarT subject happened to be reinforced $57 \%$ of the time in his/her Phase 2, 61\% in Phase 3, and 63\% in Phase 4, a yoked Prob subject would also be reinforced $57 \%, 61 \%$, and $63 \%$ during Phases 2,3 , and 4 . Because the variability contingencies were of main interest, 53 randomly selected subjects were placed in the VarT group and 18 in the Prob.

Variability threshold (Var'T) contingencies. Modifications to the VarT contingency used in Experiment $2 \mathrm{~A}$ were made, since it was thought that human subjects could discover a simple strategy to "solve" the variability contingencies applied to the rats. As a string of " 1 " and " 2 " responses was emitted on the keyboard (again, there were no "trials" apparent to the subjects, and each response resulted in assessment of whether the response met the contingency), the stored responses were divided into three levels, with Level 1 defined by sets of four consecutive responses (the current response plus the previous 3 ), Level 2 by sets of four responses each separated by one (every other response, beginning with the most recently emitted response), and Level 3 by sets of four responses, each separated by two responses (every third response). Assume, for example, the following sequence of left and right responses, in the order shown: LRRLLRRRLRLR ..., with a left response occurring first, followed by two right responses, two more left, and so on. Level 1 sets would be LRRL, RRLL, RLLR, LLRR, and so on. Level 2 sets would be LRLR, RLRR, RLRL, LRRR, and so on. Level 3 sets would be LLRR, RLRL, RRLR, and so on.

The three levels were treated independently. Counters maintained the frequencies of each possible four-response sequence and the sum, as in the rat procedure described in Experiment 2A, but now separate sets of counters were maintained for each of the three levels. On emission of a response, the appropriate counters at each of the three levels were added to $(+1)$ and evaluated. A relative frequency was computed by dividing the sequence counter by the sum counter, at each of the three levels. These relative frequencies were then compared with the threshold value. If and only if all three relative frequencies were equal to or less than the threshold, reinforcement was possible. Three levels helped us to identify repetitive, or systematic, response strategies. If all three relative frequencies met the threshold criterion, then all 51 counters ( 17 counters per level $\times 3$ levels) were multiplied by a weighting coefficient, as described in Experiment 2A.

There was one additional complexity. As indicated in Experiment $2 \mathrm{~A}$, application of a weighting coefficient enabled recent sequences to contribute more to the evaluation than earlier occurring sequences. The value of the weighting coefficient determines whether relatively long-term patterns or short-term patterns would be most easily detected. In the human case, we thought it important to try to identify short-, intermediate-, and long-term patterns concurrently; therefore, at each of the three levels, three separate weighting coefficients were applied, resulting in a total of 153 counters ( 17 counters $\times 3$ levels $\times 3$ weighting coefficients). The three weighting coefficients were $.95, .97$, and .99 . Each coefficient was associated with a separate threshold value: $0.124,0.102$, and 0.088 , respectively. Using a pseudo-random response generator, we had determined threshold values for each of the three weighting coefficients such that, at each coefficient, probabilities of the random generator being reinforced were equal. Under these contingencies, a simulating random generator was reinforced $76 \%$ of the time. These complexities were added to increase the likelihood of detecting repetitions of any patterns.

In summary, performances were evaluated on line, and a response was reinforced only if all nine currently activated relative frequencies ( 3 levels $\times 3$ weighting coefficients) had values less than the relevant thresholds. Each phase consisted of 300 responses (a phase lasting about $5 \mathrm{~min}$ ), with the first phase being Prob for all 
subjects and, for most subjects, the next three phases involving reinforcement of variability, as just described. For 18 control subjects, all four phases were Prob. The subjects' weighted response frequencies were retained across the four components. The subjects were told nothing about the contingencies or the different groups. They were told only to gain as many points as possible. Because of time limitations, few subjects answered postsession questions.

\section{Results}

Figure 5 (bottom panel) shows $U$ values for the two groups as a function of whether variability was reinforced (VarT) or not (Prob). For ease of comparison with the rats, we compared initial and final phases only. (Exactly the same pattern of results was seen when all four phases were included in the human data analyses.) The group $X$ phase interaction was statistically significant $[F(1,69)=$ $15.65, p<.0002]$. The groups did not differ during the first phase but did differ during the last $[F(1,69)=26.61$, $p<.001]$. In the Prob-VarT group, $U$ values increased significantly across the phases $[F(1,69)=14.33, p<.001]$, whereas in the Prob-Prob control group, $U$ values decreased $[F(1,69)=5.63, p<.02]$. Therefore, variability of response sequences was higher in human subjects when variability was reinforced than when not, exactly as had been the case for the rats. Again as with the rats, the effects of reinforcement on variability were shown both across phases (within subjects) and across groups.

\section{Discussion}

For both rats and people, variability increased across the session(s) when variations were reinforced, and variability decreased when reinforcement was provided probabilistically (i.e., without regard to levels of variation). The main difference between the human and rat results was that levels of variability in the human control subjects (those experiencing only probabilistic reinforcement) decreased significantly across the experiment, whereas the decrease in the control rats did not reach a significant level, but the two functions were quite similar. Thus, behavioral variability was sensitive to its consequences in both rats and people, results consistent with those from the literature (Machado, 1989; Neuringer, 1986; Page \& Neuringer, 1985; Stokes, Mechner, \& Balsam, 1999).

In neither rat nor human cases did levels of variability reach that of a random generator. When the present experiment was simulated with a computer-based random generator emitting the responses, $U$ values approached 1.0, whereas the VarT group's was less than .94. As indicated above, the random generator was reinforced on $76 \%$ of trials, whereas, during the last phase of the experiment, the human subjects in the VarT group were reinforced on $54 \%$ of their trials. Consistent with other results from the literature (Neuringer, 1986; Stokes et al., 1999), high levels of variability can be reinforced, but approximating a random distribution requires extensive training.

The procedures in Experiment $2 \mathrm{~A}$ and $2 \mathrm{~B}$ can be used to motivate a number of questions for discussion: How is it possible to reinforce variability - that is, what is the unit on which reinforcement exerts its control (see Machado,
$1992,1997)$ ? Why is probabilistic reinforcement a necessary control procedure? When is variability functional, and under what circumstances is it reinforced (e.g., in avoiding predation, locating new sources of food [foraging], in problem solving, and when engaging in creative acts)?

Broader issues may also be broached. According to $\mathrm{Zu}$ riff (1985), the goal of behavioral research is prediction and control. However, when contingencies of reinforcement require high variability, prediction and control may be difficult or impossible. Reinforced variability may also be relevant to an explanation of voluntary behavior. Voluntary implies that a behavior is potentially unpredictable and, at the same time, potentially explainable by the contingencies, as was the case in Experiments 2A and 2B.

\section{GENERAL DISCUSSION}

Introductory psychology students, mainly freshmen and sophomores, listened to seven 90 -min lectures on basic behavioral topics and participated in eight $50-\mathrm{min}$ laboratory periods, two per week. Each pair of students trained an experimentally naive Long-Evans rat. Four laboratory periods are described in this paper.

In the first of these, the students studied responding by rats under concurrent FI-FI schedules of reinforcement, with the ratio of the FI intervals constant across the session. There were, however, four different ratios, randomly allocated across the rats. The main finding was that when the 40 rats were combined for purposes of analysis, relative frequencies of choosing left and right levers were linearly related to relative frequencies of obtained reinforcement, a matching-like function. Although the data were collected in a single session, and the function was obtained by combining across subjects, the results were orderly and were consistent with those in the literature (Davison \& McCarthy, 1988).

In the next two laboratory periods, the rats were first reinforced probabilistically for emitting four-response sequences across the two levers, with the variability (or repetitiveness) of the sequences not mattering for reinforcement. In the second session, high levels of sequence variability were required (for most rats) for reinforcement (with the other rats continuing on the "probabilistic" reinforcement as controls). The main finding was that sequence variability increased significantly when variability was reinforced. Again important for instructional use, significant results were obtained rapidly, with results similar to those in the literature (Page \& Neuringer, 1985).

During the next session, the students served as subjects in two computer "game" procedures. In one, responses on the computer keyboard were reinforced according to a concurrent schedule of reinforcement. In the other, variable sequences were reinforced. In both, points (as reinforcers) were accumulated during the game period, with the highest scoring students being invited to the instructor's mountain cabin for fun and food.

Most important for instructional purposes was the parallel nature of the laboratory procedures. The same 
questions were asked of rats pressing levers for pellets in operant chambers and of students pressing keys on a computer keyboard for points. Others have also found it useful to compare human performances in computer games with animal performances in operant chambers (Tustin, 1994; Washburn \& Gulledge, 1995). The parallel nature of the results enabled students better to appreciate the influences that consequences exert on behaviors, with respect both to choices (McDowell, 1989) and to behavioral variability (Page \& Neuringer, 1985). Furthermore, the students were led to compare their own thoughts with the objective reinforcement contingencies. Which was more closely correlated to their distribution of responses? This last question is relevant to the general issue of learning without awareness. The students' thoughts about causal relationships were generally incorrect. Said differently, choices were controlled by the reinforcement contingencies despite the fact that the students misidentified the controlling contingencies. The term superstitious could be applied to many of the student descriptions (Vyse, 1997).

We conclude with four lessons we learned that might be useful to other instructors. First, when students compare their own operant responding with that of a rat, they come better to appreciate the relevance to human behavior of animal models. Second, both animal and human procedures were designed to generate large quantities of data in very limited time periods. Only by modifying common procedures was this goal attained. Third, when studying human operant behavior, there is an opportunity to obtain subjective estimates of contingencies and responses and to relate these to environmental events. By directly comparing cognitions and contingencies, the instructor can bridge two often separate fields of study. Lastly, computer "games" are useful for teaching psychological and behavioral principles: Students experience firsthand the phenomena about which they read (see Case, 1995; Donchin, 1995). Such firsthand experiences provide a level of intuitive understanding otherwise hard to obtain.

\section{REFERENCES}

AllaN, R. W. (1995). The matching game: Testing for the generality of the matching law. Behavior Research Methods, Instruments, \& Computers, 27, 206-210.

BAUM, W. M. (1974). On two types of deviation from the matching law: Bias and undermatching. Journal of the Experimental Analysis of Behavior, 22, 231-242.

CASE, D. A. (1995). On trekking to operant frontiers. Behavior Research Methods, Instruments, \& Computers, 27, 211-216.

DAVISON, M., \& MCCARTHY, D. (1988). The matching law: A research review. Hillsdale, $\mathrm{NJ}$ : Erlbaum.

Denney, J., \& Neuringer, A. (1998). Behavioral variability is controlled by discriminative stimuli. Animal Learning \& Behavior, 26, 154-162.

DonchIN, E. (1995). Video games as research tools: The Space Fortress game. Behavior Research Methods, Instruments, \& Computers, 27, 217-223.

FINDLEY, J. D. (1958). Preference and switching under concurrent scheduling. Journal of the Experimental Analysis of Behavior, 1 , 123-144.

Gallistel, C. R. (1990). The organization of learning. Cambridge, MA: MIT Press.

HeRrNSTEIN, R. J. (1961). Relative and absolute strength of response as a function of frequency of reinforcement. Journal of the Experimental Analysis of Behavior, 4, 267-272.

Kollins, S. H., Newland, M. C., \& CRITChfield, T. S. (1997). Human sensitivity to reinforcement in operant choice: How much do consequences matter? Psychonomic Bulletin \& Review, 4, 208-220.

MACHADO, A. (1989). Operant conditioning of behavioral variability using a percentile reinforcement schedule. Journal of the Experimental Analysis of Behavior, 52, 155-166.

MACHADO, A. (1992). Behavioral variability and frequency-dependent selection. Journal of the Experimental Analysis of Behavior, 58, 241263.

MACHADO, A. (1997). Increasing the variability of response sequences in pigeons by adjusting the frequency of switching between two keys. Journal of the Experimental Analysis of Behavior, 68, 1-25.

MAZUR, J. E. (1998). Choice and self-control. In K. A. Lattal \& M. Perone (Eds.), Handbook of research methods in human operant behavior: Applied clinical psychology (pp. 131-161). New York: Plenum.

MCDowell, J. J. (1989). Two modern developments in matching theory. Behavior Analyst, 12, 153-166.

Neuringer, A. (1986). Can people behave "randomly"?: The role of feedback. Journal of Experimental Psychology: General, 115, 62-75.

PAGE, S., \& NeURINGER, A. (1985). Variability is an operant. Journal of Experimental Psychology: Animal Behavior Processes, 11, 429-452.

RaCHLIN, H. (1989). Judgment, decision and choice: A cognitive/behavioral synthesis. New York: Freeman.

ShIMP, C. P. (1971). Matching in a concurrent FI FI schedule. Psychonomic Science, 22, 27-28.

Stokes, P. D. (1999). Learned variability levels: Implications for creativity. Creativity Research Journal, 12, 37-45.

Stokes, P. D., Mechner, F., \& Balsam, P. D. (1999). Effects of different acquisition procedures on response variability. Animal Learning \& Behavior, 27, 28-41.

TUSTIN, R. D. (1994). Preference for reinforcers under varying schedule arrangements: A behavioral economic analysis (Special issue: Integrating basic and applied research). Journal of Applied Behavior Analysis, 27, 597-606.

VySE, S. A. (1997). Believing in magic: The psychology of superstition. New York: Oxford University Press.

Washburn, D. A., \& Gulledge, J. P. (1995). Game-like tasks for comparative research: Leveling the playing field. Behavior Research Methods, Instruments, \& Computers, 27, 235-238.

WiLliams, B. A. (1988). Reinforcement, choice, and response strength. In R. C. Atkinson, R. J. Herrnstein, G. Lindsey, \& R. J. Luce (Eds.), Stevens' Handbook of experimental psychology (pp. 167-244). New York: Wiley.

ZURIFF, G. E. (1985). Behaviorism: A conceptual reconstruction. New York: Columbia University Press.

\section{NOTE}

1. An extreme example occurs when every choice is reinforced on each of the alternatives (e.g., FR 1-FR 1). Under such conditions, the obtained rates of reinforcement are directly controlled by the emitted responses.

(Manuscript received June 18, 1999; revision accepted for publication January 26, 2000.) 